\title{
Injection Molding Technique for Fabrication of Flexible Prosthesis from Flexible Thermoplastic Denture base Materials
}

\author{
Kunwarjeet Singh, Nidhi Gupta
}

\section{ABSTRACT}

Purpose: To know properties, step-by-step procedure for fabrication and insertion of flexible prosthesis.

Background: Flexible denture base materials were introduced to dentistry by the name of Valplast and Flexiplast in 1950's. Injection molding technique is used for fabrication of various types of prostheses from these materials and fluid resins. Pryor used injection molding technique for introducing unpolymerized acrylic resin into the mold. In the mid 1970's Ivoclar introduced an injection molding system which used an acrylic resin modified for the injection molding process. Recently, numbers of dental manufacturing companies have introduced injection molding systems.

Materials and methods: The flexible denture base materials
are superpolyamides which are available in the form of granules
in cylinders of different sizes. These materials are thermoplastic
in nature and needed to be converted into fluid form before
pouring into mold under pressure. Each cylinder should be
plasticized for 15 to 20 minutes at 550 to $560^{\circ} \mathrm{F}$ in an electric
cartridge furnace before injecting the material into the flask.
While injecting, the cylinder should be aligned with opening of
flask and the levers of the press should be turned rapidly to
apply firm pressure until the springs of the press are fully
compressed. The pressure should be maintained for 3 to 5
minutes and the flask should be allowed to bench cool for 15 to
20 minutes before opening.

Conclusion: This technique can be used for fabrication of differenttypes of prostheses from flexible denture base materials and fluid resins.

Keywords: Injection molding technique, Flexible partial dentures, Valplast, Flexiplast, Nylon, R etento-Grip tissue bearing technique, Superpolyamides.

How to cite this article: Singh K, Gupta N. Injection Molding Technique for $\mathrm{F}$ abrication of Flexible Prosthesis from Flexible Thermoplastic Denture base Materials. World J Dent 2012;3(4):303-307.

\section{Source of support: Nil}

\section{Conflict of interest: None}

\section{INTRODUCTION}

Flexible denture base materials are thermoplastic material which becomes fluid when heated in an el ectrical cartridge furnace to a specific temperature. These are available in the form of granules in cartridges of different sizes (Fig. 1). Small size cartridges are used for fabrication of small size flexible partial dentures and large size cartridges are used

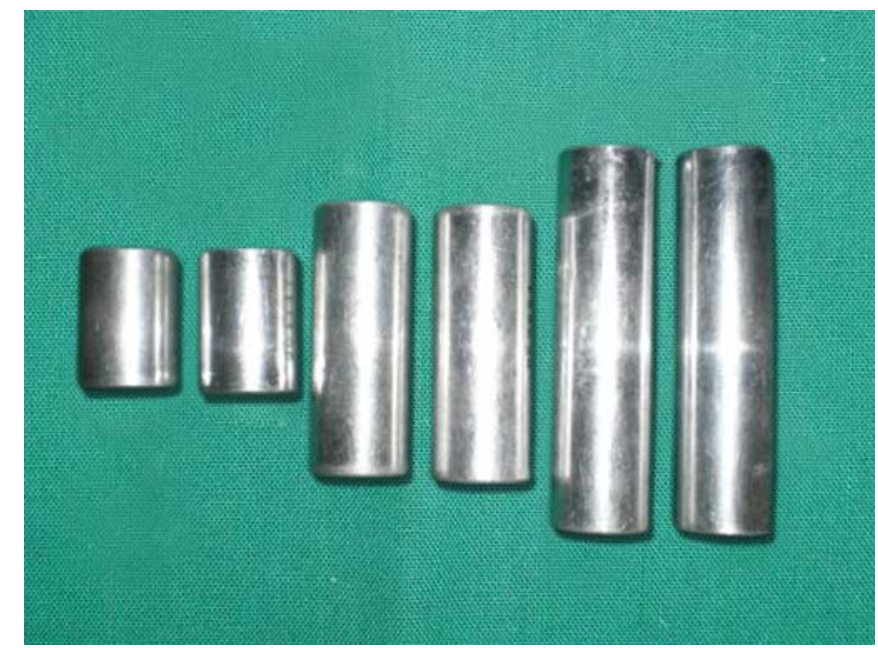

Fig. 1: Cartridges containing granules of flexible denture base material

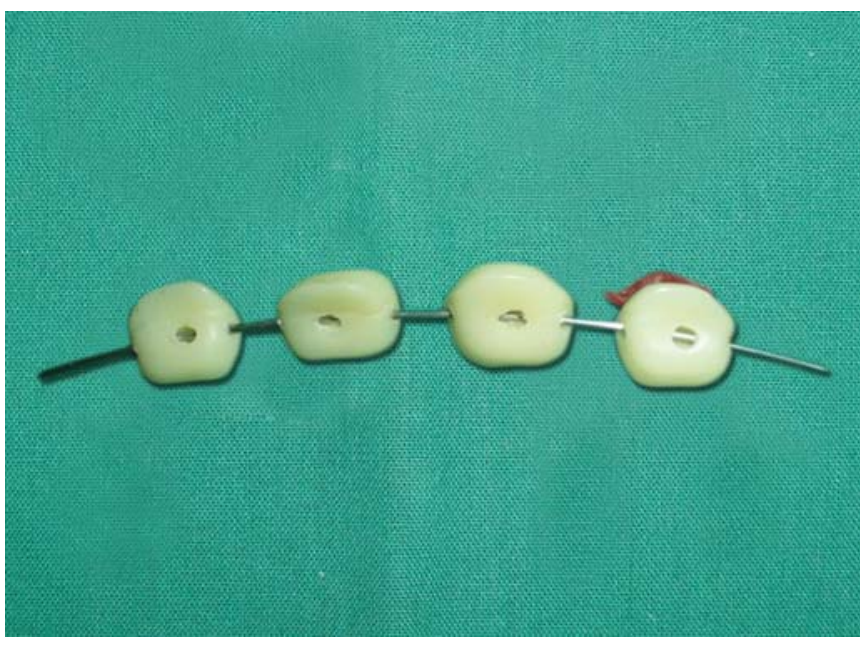

Fig. 2: Wire showing orientation of holes

for large size removable partial dentures (RPDs) and removable flexible complete dentures. These materials are first introduced to dentistry in 1956 by the name of $V$ al plast and Flexi plast. ${ }^{1,2}$ These are superpolyamides which belong to nylon family which has inherent property of flexibility. ${ }^{1}$ The flexibility of the prosthesis also depends on the thickness of the prosthesis which should be less than $2 \mathrm{~mm} .{ }^{1}$ The prosthesis has very good retention. Thin finger like extension of the material into undercuts acts as a clasp and helps in pressureless retention of the prosthesis. The clasps fabricated from the same material provide much better esthetics. Flexible denture base material can also be used 
in combination with cast partial metal framework where clasps and saddle can be made from flexible material, thereby improving the esthetics and major connector and rest from the metal. ${ }^{1}$ These are monomer free nonallergic materials.

The acrylic teeth do not bond chemically with flexible denture base. Teeth are mechanically retained by making ' $T$ ' shape holes in the acrylic teeth (Fig. 2) into which fluid flexible denture base material flow during injection of fluid resin into the mold. Once the resin sets inside the holes, it retains the teeth with denture base.

This article describes a pressure molding technique for fabrication of flexible prosthesis.

\section{FABRICATION/LABORATORY PROCEDURE}

1. M outh preparation is usually not required for fabrication of flexible partial dentures as required for removable cast partial denture. Just make an impression with hydrocolloids or elastomeric impression material and obtain a master cast. Duplicate the master cast in any suitable duplicating material. M ake wax occlusal rim, record jaw relations and mount the casts on the articulator.

2. Make ' $T$ ' shape holes (diatorics) in teeth of selected shade. The orientation of the holes is show $n$ in Figure 2. The holes are made for mechanical retention of acrylic teeth to flexible denture base. The holes may be made before arranging teeth or removing the teeth from the mould after dewaxing. The wax should be completely removed from the holes in the teeth. If wax is not completely removed from holes, flexible material may not flow properly into holes from the cartridge (Fig. 1), thereby affecting the retention of teeth with denture base. This technique of retention of acrylic teeth with flexible denture base is known as Retento-Grip tissue bearing technique. This prosthesis is contraindicated in patients where interarch space is less than $4 \mathrm{~mm}$ (insufficient space for placing diatorics).

3. A rrange the teeth, do the try in and wax up and attach the sprue formers to make the channels for flowing of fluid resin into mold. Prefabricated sprue formers are available but these can al so be made from modeling wax.

4. A fter investing in a special flask designed for injectionmolding technique (Fig. 3), dewaxing is done by placing flasks in boiling water for 3 to 5 minutes to soften the wax. 0 pen the flask and flush with clean boiling water to remove all the residue of wax. Check the flask margin and ensure that both flask halves fit together with intimate metal contact. A pply a thin coat of separating agent to model and allow the model to dry completely.

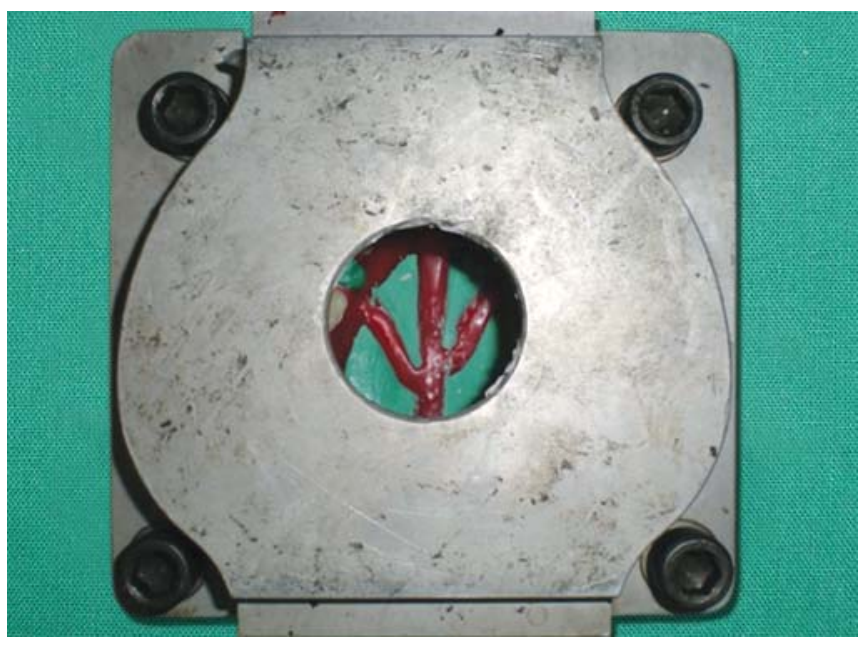

Fig. 3: Injection molding flask
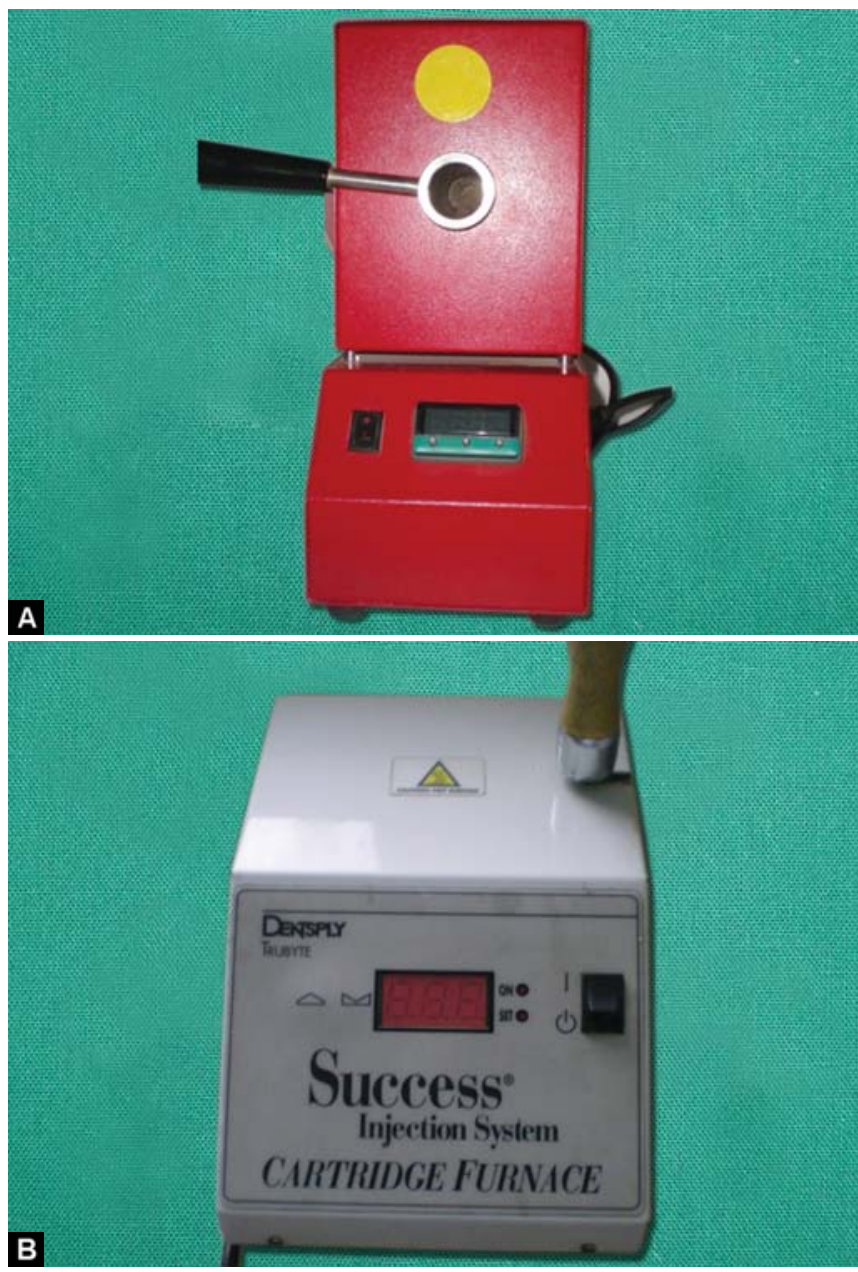

Figs 4A and B: Electric cartridge furnace

5. Select a cartridge of suitable size (Fig. 1) and spray silicone spray on it. Place it in cartridge carrier which is then placed in electric cartridge furnace (Figs 4 A and B) used for softening of flexible denture base material. The application of spray prevents adhesion of cartridge with cartridge carrier and allows smooth separation. The 


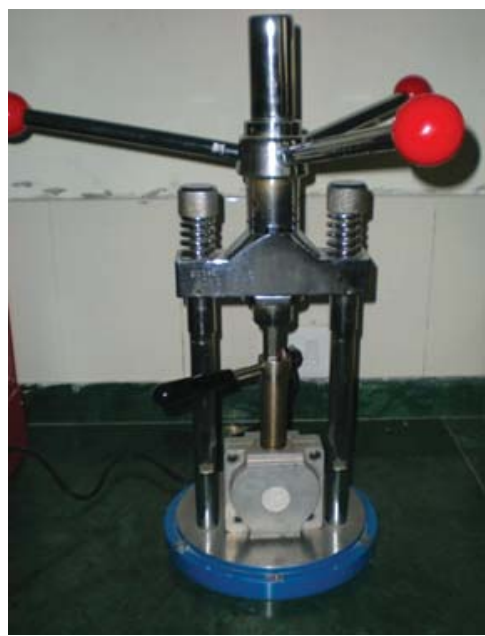

Fig. 5A: Manual compression unit

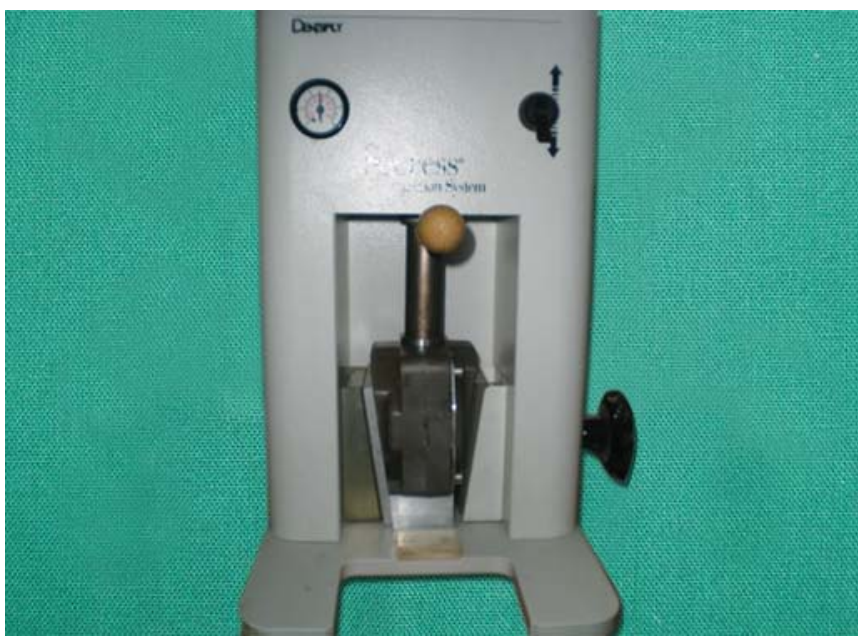

Fig. 5B: Pressure compression unit

material should be plasticized for 15 to 20 minutes at 550 to $560^{\circ} \mathrm{F}$ (V al plast). The softening temperature may be different for different types of flexible denture base material (follow manufacturer instructions). Maintain this temperature for 15 to 20 minutes. Remove the cartridge from the electric furnace and place it on the inlet of the flask and compress the mechanical compressor (Fig. 5A). The time between removing the cartridge assembly from the furnace and injection should be less than 1 minute. If longer, the cartridge will begin to cool and may result in partial or no injection. The levers of the press should be turned rapidly to apply firm pressure until the springs of the press were fully compressed. ${ }^{3}$ The pressure should be maintained for 3 to 5 minutes. The pressure was then relieved and the flask was allowed to bench cool for at least 15 to 20 minutes before opening. ${ }^{3}$ The material flows through the sprues into the mold. Open the flask and retrieve the prosthesis. The flasks should not be opened immediately to prevent distortion of the prosthesis.

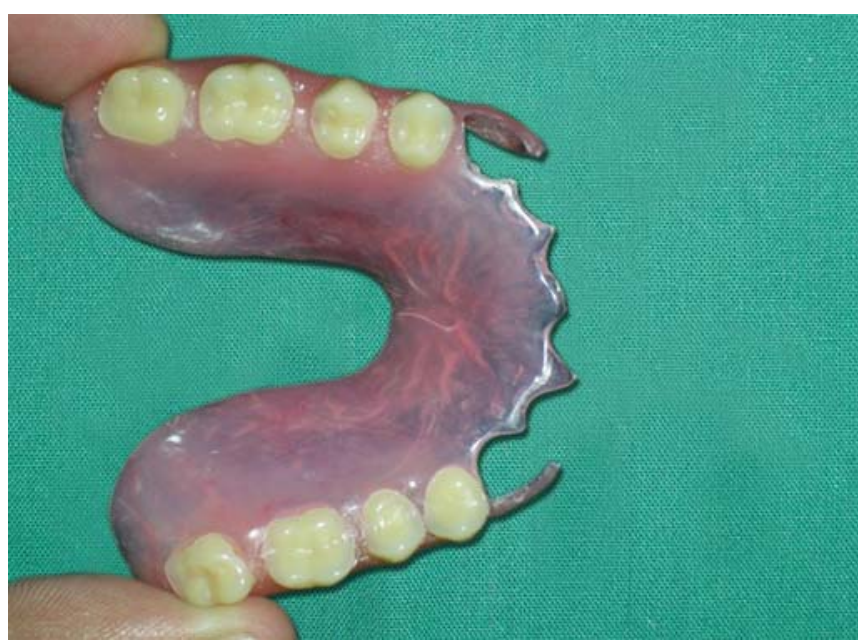

Fig. 6: Flexible RPD

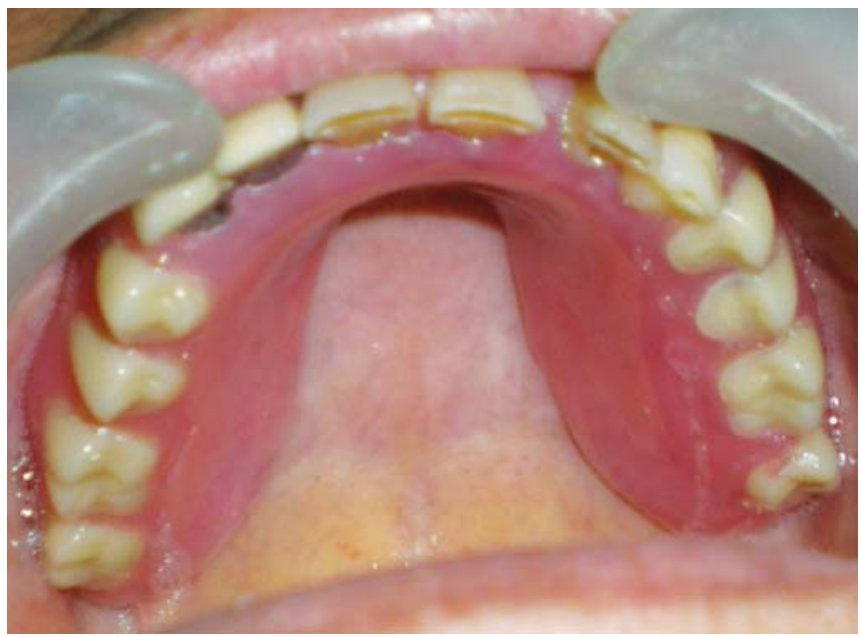

Fig. 7: Prosthesis in patient mouth

If success injection system (Fig. 5B) is used, the injection pressure should be 100 psi (minimum 75 psi) and injection time should be 1 minute.

6. A fter retrieving the prosthesis, finish the prosthesis. The finishing procedure used for flexible partial denture is different from finishing of acrylic resin prosthesis. The acrylic instruments should not be used because they generate heat and cause fiber formation and roughness of the prosthesis. B eing thermoplastic material, the high heat generated while finishing with acrylic trimmers may soften and distort the prosthesis. The sprue formers are cut with special type of knife or disk and finishing is done with valcinate burs and green and pink mounted stones, usually used for porcelain finishing, using a rapid and light shaving motion.

7. The staining resistance of the prosthesis depends on its shiny/lustrous appearance. The surface of prosthesis should be highly shiny (Figs 6 and 7) without any roughness to resist the staining. The polishing of flexible partial dentures is done in different steps after cutting 


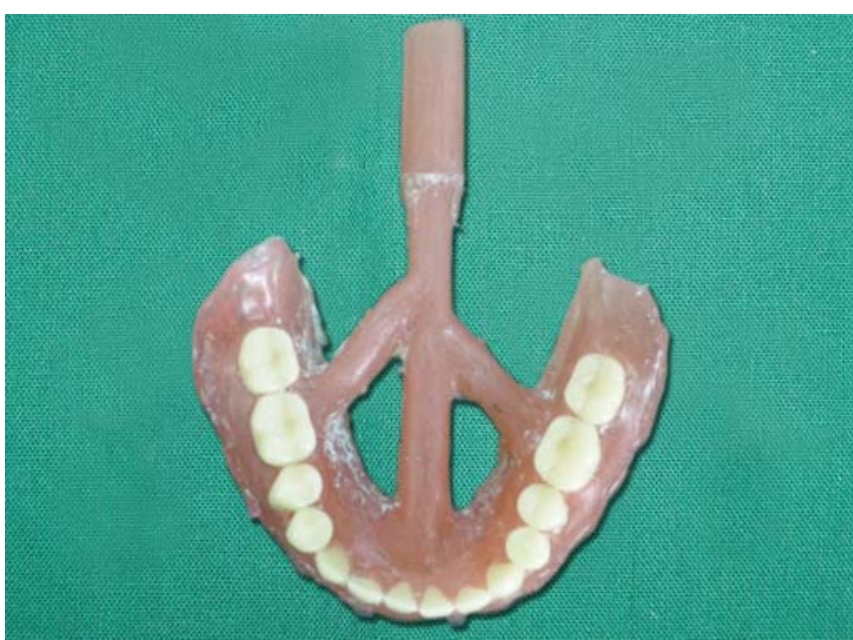

Fig. 8: Mandibular complete flexible denture with sprue former

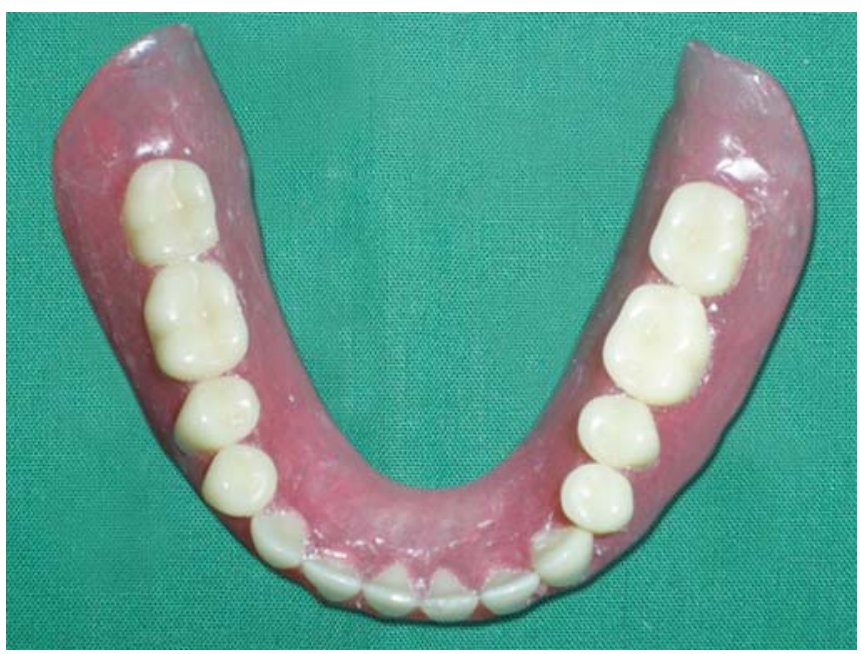

Fig. 9: Finished and polished flexible mandibular complete denture

the sprues (Fig. 8) and finishing. The first step is polishing with pumice or pumice substitute. Mix the pumice with water, place on prosthesis and polish with the help of buff. Second step is polishing with brown Tripoli which is also used for polishing of gold and acrylic. The Tripoli develops shine on the prosthesis and also little heat generated during polishing seals the surface to resist discoloration and staining. The prosthesis should dip in cool water while polishing with Tripoli to avoid warping or scalding of the surface. The Tripoli oil residue can be removed from the prosthesis with soft bristle denture brush. To maintain the shine of the prosthesis, it is important to clean the prosthesis daily after every meal.

Finally, high luster shine is achieved by polishing with polishing cake. Polishing cake is used with dry buff to develop highly bright/luster surface. Figures 7 and 9 shows polished flexible maxillary RPD and flexible mandibular complete denture respectively.

\section{INSERTION TECHNIQUE}

Flexible prosthesis must be placed in hot boiling water for about 1 minute prior to insertion in patient's mouth. Remove and allow it to cool just to the point where it can be tolerated by the patient. This process makes the partial denture as flexible as it would be at body temperature. Gently insert the prosthesis in patient's mouth. The hot water treatment permits a very smooth initial insertion and a good adaptation with the natural tissues in the mouth. If patient senses any discomfort because of the tightness of a clasp, the clasp may be loosened slightly by immersing that area of the clasp in hot water and bending the clasp outward. If a clasp required tightening, the clasp area is immersed in hot water and bent inward to tighten.

A fter insertion of prosthesis (Fig. 7), instruct the patient to remove the prosthesis during night and keep it in water. Remove the prosthesis during brushing of remaining natural teeth because toothbrush may produce scratch on the prosthesis. Clean the prosthesis after every meal.

\section{DISCUSSION}

Thermoplastic materials for dental prostheses were first introduced to dentistry in the 1956 by the name of $\mathrm{V}$ al plast (Valplast Int Corp, USA) and Flexiplast (B redent, Germany). ${ }^{1,2}$ Different types of prosthesis can be fabricated from these materials by injection molding technique. ${ }^{2}$ Injection-molding technique is used for fabrication of flexible denture base prosthesis. In the mid 1970s, Ivoclar (Schaan, Liechtenstein) introduced an injection molding system ${ }^{4}$ that used an acrylic resin modified for the injectionmolding process. Recently, a number of dental manufacturing companies have introduced injectionmolding systems. Fluid resin flows into the mold cavity through sprues formed by sprue formers. The same technique is used for relining and rebasing of flexible partial dentures. The reline and rebasing are less frequent with flexible partials than with acrylic and metal partials. A s long as the mouth undergoes slow, healthy, gradual change, the flexible partial will remain functional and not require relining or rebasing until drastic abnormal changes takes place in the mouth.

The rest or vertical stop is not required with flexible partial dentures because under functional loading, the flexible partial flex and exert undesirable stresses on abutment teeth. The rest is only useful with rigid framework to transfer load to abutment tooth properly.

Selection of the cartridge containing flexible denture base material is very important to avoid any shortfall/ deficiency, which is possible but difficult to rectify. It is selected on the basis of type and size of the prosthesis. For 
Injection Molding Technique for Fabrication of Flexible Prosthesis from Flexible Thermoplastic Denture base Materials

partial dentures, usually small size cylinder is selected but if partial is of large size then large size cylinder should be used. This material is costly as compared to acrylic resins.

The rapid and firm pressure should be applied during injection of soft fluid resin into mold until the spring of the press is fully pressed. This pressure should be maintained for 3 to 5 minutes so that material properly flows into all parts of the mold. ${ }^{3} \mathrm{~A}$ fter releasing pressure, the flask should be bench cool for 15 to 20 minutes before retrieving the prosthesis. $^{3}$

The disadvantage of injection-molding technique is the high cost of the equipments required for fabrication of prosthesis from the thermoplastic resins. The specially designed equipments required for this technique includes mechanical compress, investing flask, cartridges, cartridge carrier and electric furnace. A nother disadvantage is that it requires firm and quick application of the pressure for proper flow of the resin through sprues into the mold. If pressure is not applied quickly, material will not flow properly into the mold and results in incomplete fabrication of the prosthesis.

\section{CONCLUSION}

Injection/pressure-molding technique can be used for the fabrication of flexible denture base resin prosthesis, such as RPDs, N esbit bridge (unilateral RPDs) complete dentures, maxillofacial prosthesis, space maintainer, TM J splints, gum veneers, night guard. Depending upon size and type of prosthesis, selection of cylinder (small or large) containing flexible denture base material for fabrication of a any of above prosthesis and application of rapid and firm pressure are important for success of this technique.

\section{REFERENCES}

1. Stern M N. V alplast flexible partial dentures. New Y ork State Dent J 1964 Feb;30:123-36.

2. N egrutiu $M$, Sinescu $C$, Romanu $M$, et al. Thermoplastic resin for flexible framework removable partial dentures. TM J 2005;55:295-99.

3. Parvizi $A$, Lindquist $T$, Schneider $R$, et al. Comparison of the dimensional accuracy of injection molded denture base materials to that of conventional pressure-pack acrylic resin. J Prosthodont 2004 J un;13(2):83-89.

4. Keenan PL, Radford DR, Clark RK. Dimensional change in complete dentures fabricated by injection molding and microwave processing. J Prosthet Dent 2003 J an;89(1):37-44.

\section{ABOUT THE AUTHORS}

\section{Kunwarjeet Singh (Corresponding Author)}

Reader, Department of Prosthodontics, Institute of Dental Studies and Technologies, M odinagar,UttarPradesh, India, e-mail: drkunwar@gmail.com

\section{Nidhi Gupta}

Reader, Department of Pedodontics, ITS Dental College M urad N agar, Uttar Pradesh, India 